\title{
Pearls in paediatric trauma neuroimaging reporting - What not to miss
}

\author{
Authors: \\ Raksha Ramlakhan ${ }^{1}$ \\ Nicky Wieselthaler ${ }^{2}$ \\ Affiliations: \\ ${ }^{1}$ Division of Radiology, Groote \\ Schuur Hospital, South Africa \\ ${ }^{2}$ Division of Radiology, \\ Red Cross War Memorial \\ Children's Hospital, \\ South Africa \\ Correspondence to: \\ Raksha Ramlakhan \\ Email: \\ docrakshar@gmail.com \\ Postal address: \\ Private Bag, Observatory, \\ Cape Town 7937, \\ South Africa \\ Dates: \\ Received: 27 May 2015 \\ Accepted: 11 Aug. 2015 \\ Published: 03 Nov. 2015 \\ How to cite this article: \\ Ramlakhan R, Wieselthaler \\ N. Pearls in paediatric trauma \\ neuroimaging reporting - \\ What not to miss. S Afr J \\ Rad. 2015;19(2); Art. \#852, \\ 6 pages. http://dx.doi. \\ org/10.4102/sajr.v19i2.852

\section{Copyright:} \\ C 2015. The Authors. \\ Licensee: AOSIS \\ OpenJournals. This work is \\ licensed under the Creative \\ Commons Attribution \\ License.
}

Read online:

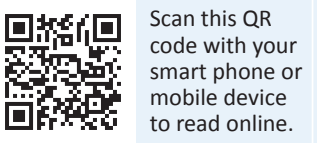

The features of paediatric head trauma are well described. This article aims to highlight review areas and 'do not miss' lesions over and above the known and easily recognised injuries often sought when analysing computed tomography (CT) brains for head injury. Particular attention is paid to fractures and three-dimensional (3D) imaging. Emphasis is also placed on bridging vein thrombosis, retroclival hematomas and corpus callosum injuries.

\section{Introduction}

Paediatric head trauma is a major cause of mortality and morbidity. A review of the Red Cross Children's Hospital 2013 trauma statistics showed that of the approximately 1570 patients seen for a 'head injury', more than half of these were due to a fall. The other half were attributed to motor vehicle or pedestrian vehicle accidents (either high or low impact) or miscellaneous causes. Approximately $4 \%$ of head injuries were due to assault.

Approximately 1000 CT scans for head trauma are performed per year, indicating that about twothirds of patients who present with head trauma have at least one head CT. A large proportion of these scans are normal. However, in the case of the abnormal scans, it is the radiologist's role to accurately recognise all injuries in order to allow the clinician to determine an appropriate management protocol.

Extensive articles have been written on paediatric head trauma, but over the years we have encountered some areas that require special review. We would like to highlight some 'do not miss lesions', as well as give some 'tips' as to which 'lesions' to image or investigate further, because missing them may be detrimental to the paediatric patient.

\section{The pursuit of fractures}

Skull fractures are a marker of severity of injury to a child's cranium. Fractures provide clues to localising the site of impact of an injury and assist in predicting and understanding the associated intracranial consequences.

Calvarial fractures are a sign of primary brain injury in both accidental and non-accidental trauma. The presence of fractures in both clinical settings therefore has a significant impact on further and future aspects of management. ${ }^{1}$

\section{Use of the 3D reconstruction algorithm For fracture detection}

The availability of 3D reconstructions for CT makes this an invaluable problem-solving, postprocessing tool. The 3D reconstructions are particularly useful in resolving fractures that are not easily identifiable in the axial imaging plane on CT (Figure 1a and Figure 1b). ${ }^{1}$ Where there is confusion, or poor knowledge of suture anatomy, it is useful to use the other side for comparison (Figure 1c).

The 3D application must be a routine and perhaps an initial step when evaluating a CT head scan, irrespective of whether the scan is performed for a 'medical' indication or in the setting of trauma. Non-accidental injury (NAI) is commonly seen at our institution, and the radiologist is often the first and initial clinician to detect these cases, especially when the history of abusive trauma is absent.

CT head scans performed for seizure investigation, without an accompanying history of trauma or abuse, have often led to the findings of skull fractures when the 3D setting is used. This point is emphasised by a patient who was recently referred for an urgent CT to investigate seizures. The CT scan showed a large subacute left temporal intracerebral haematoma and a left occipitoparietal subdural haematoma (Figure 2a). 

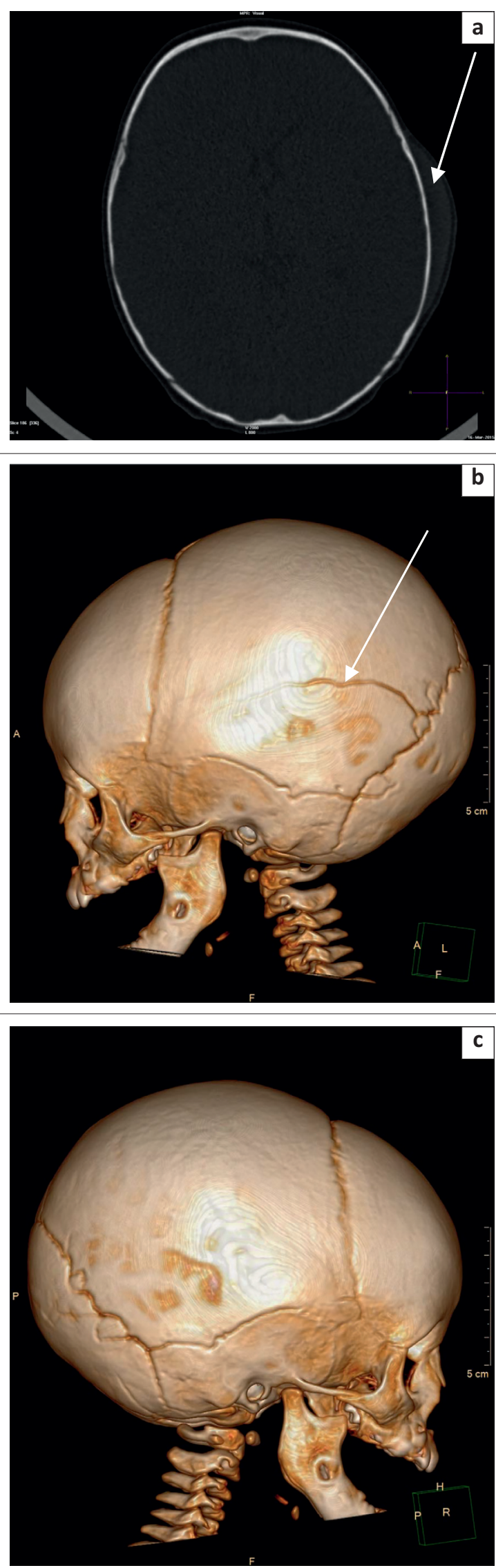

FIGURE 1: (a) An 8 month old infant fell off the bed and presented with left sided scalp swelling. Axial CT bony algorithm demonstrated left parietal scalp swelling (white arrow). No distinct fracture was visualised in this plane; (b) 3D reconstruction demonstrated a linear left parietal fracture (white arrow) (c) 3D reconstruction of the non-fractured side revealed normal sutures.
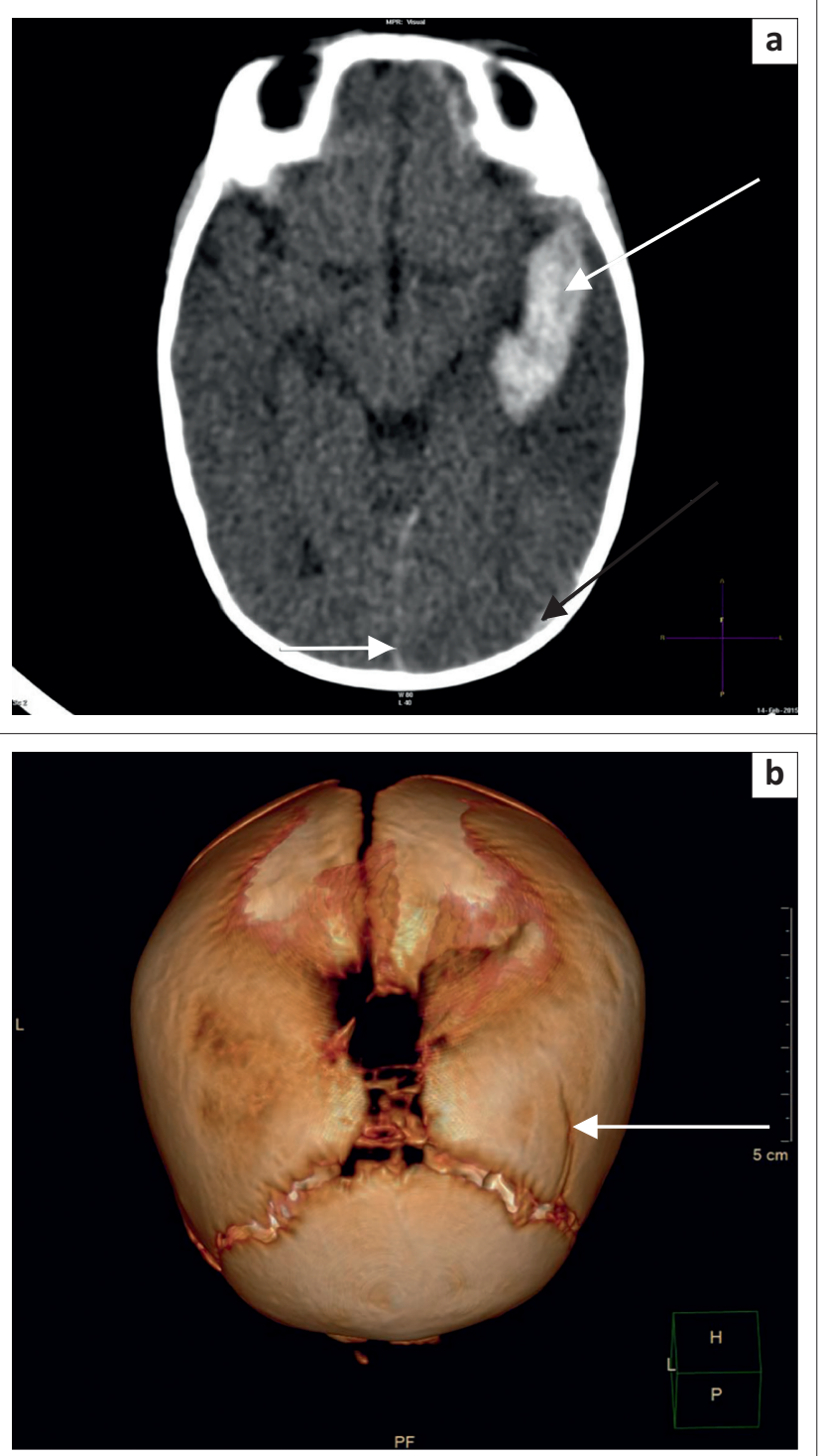

FIGURE 2: (a) A 2 month old male infant was referred with a history of focal seizures and suspected blindness. Axial CT brain demonstrated a large left temporal intracerebral haematoma (left white arrow). In addition there was a small acute left parieto-occipital subdural haematoma (black arrow) and blood in the posterior interhemispheric fissure (right white arrow). (b) 3D reconstruction demonstrated a contralateral parietal linear fracture (white arrow).

Upon evaluation of the bones on the 3D setting, it was immediately evident that there was a skull fracture on the opposite side involving the right parietal bone (Figure $2 b$ ). The referring clinician was then immediately informed and alerted to NAI as the aetiology for the haemorrhage.

\section{For suture assessment}

A knowledge of normal suture anatomy and appearance is important to the radiologist interpreting head CT. Normal sutures have a zig-zag appearance, and sclerotic borders. ${ }^{1}$

The use of 3D reconstruction provides a quick-and-easy tool with which to assess sutures, and especially asymmetry, when evaluating sutural diastasis (Figure 3). According to Bhatt et al., 'the presence of diastasis is the most common of paediatric skull fractures'. ${ }^{1}$ But in our population group, linear and depressed skull fractures were found to be more common. 


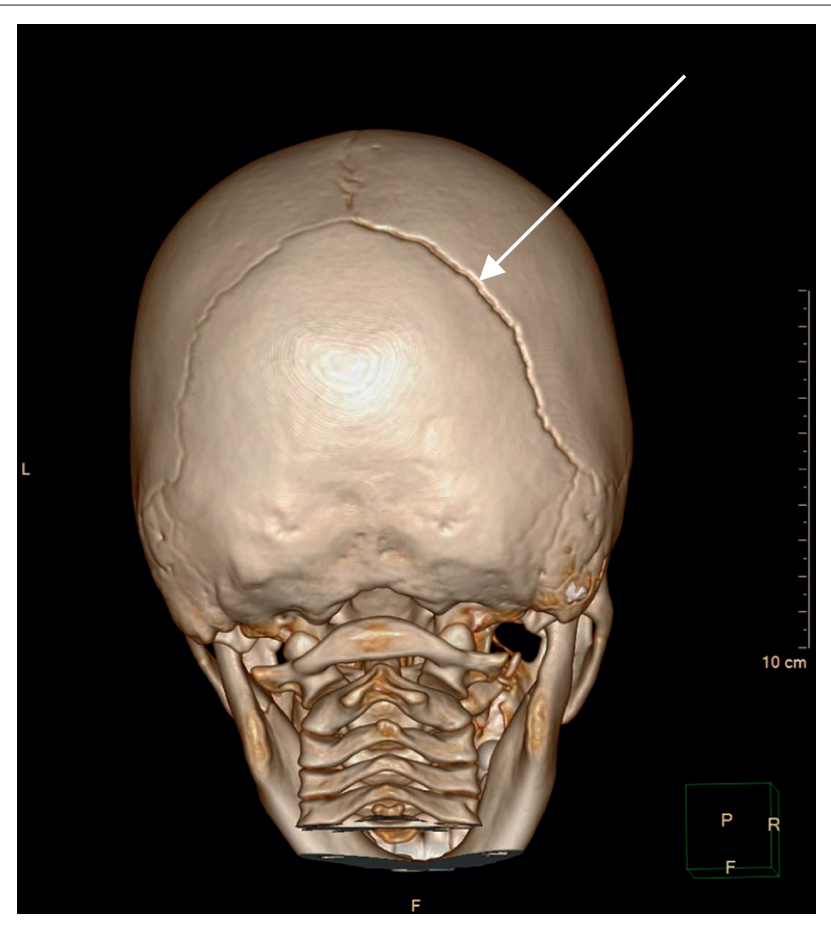

FIGURE 3: A 7 year old pedestrian was hit by a vehicle. 3D reconstruction demonstrated right lambdoid suture diastasis.

\section{Additional clues to finding fractures}

Calvarial fractures almost always occur with associated overlying soft tissue swelling. In the absence of soft tissue swelling, however, the presence of gas locules or fluid within an aerated space such as the paranasal sinuses and the mastoid air cells is a clue to the presence of localised fractures. ${ }^{1}$ Mastoid air cells are important review areas that help the radiologist not to miss the base-of-skull fractures (Figure 4).

\section{Use of coronal reconstruction for mandibular fracture detection}

Mandibular fractures are common in children and are the second most common type of facial bone fracture, following the nasal bones. ${ }^{2}$ The importance of reviewing the mandibular condyles has previously been highlighted in this journal. ${ }^{2}$ Mandibular condyle fractures can be subtle, and easily overlooked in the axial imaging plane. It is thus imperative to review thoroughly the temporomandibular joints and the upper mandible in the coronal plane (Figure $5 a$ and Figure $5 b$ ).

\section{Carotid canal fractures and carotid artery dissection}

Post-traumatic carotid artery dissection (PTCAD) in children results from rapid deceleration-type injuries to the head and neck. It is a common cause of paediatric acute arterial ischaemic stroke (AIS). ${ }^{3}$ Neurological sequelae can be variable, even devastating, depending on the degree and extent of injury. PTCAD most commonly involves the distal cervical segment of the internal carotid artery before its entry into the carotid canal at the skull base. ${ }^{3}$

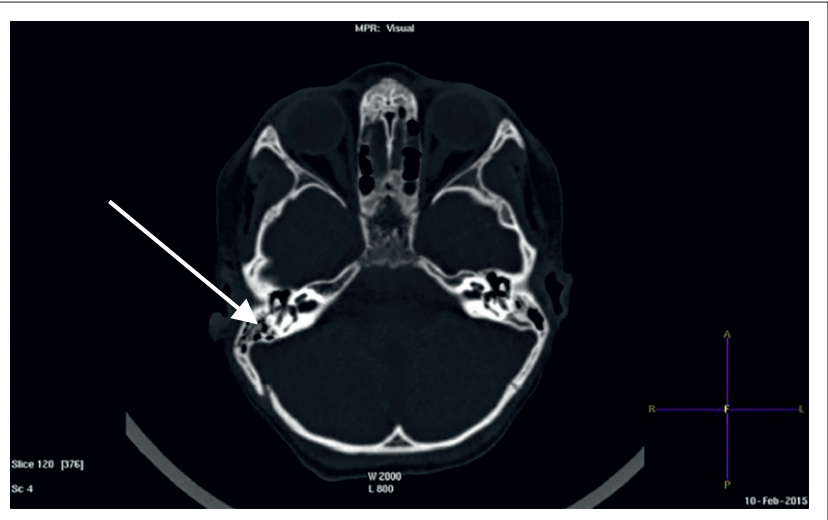

FIGURE 4: A 6 year old boy presented with a closed head injury and right otorrhea, following a fall from a moving vehicle. Axial CT bony algorithm demonstrated partially opacified right mastoid air cells. A subtle linear fracture was seen traversing the right mastoid temporal bone (white arrow).
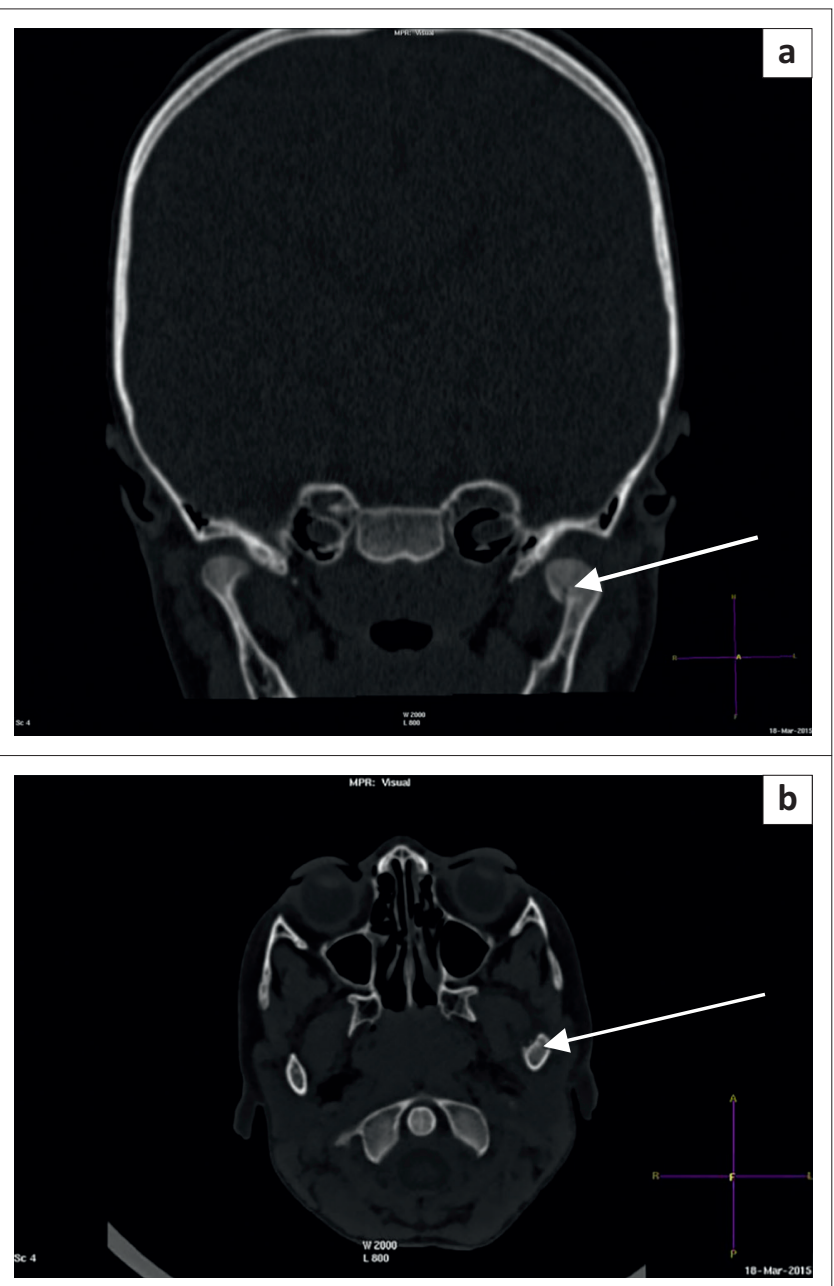

FIGURE 5: (a) A 6 year old female sustained a head injury of unknown mechanism and presented with vomiting. Coronal MPR view of the bony algorithm demonstrated a linear fracture of the left mandibular condyle (white arrow). The corresponding axial views are far more subtle; (b) Axial CT bony algorithm for the above patient, showing lucency of the left mandibular condyle (white arrow).

Non-contrasted CT head is useful for identifying base-of-skull fractures, which are associated with PTCAD. The presence of base-of-skull fractures must prompt the radiologist to review the carotid canals for fractures. Air extending along the carotid canal, in combination with base-of-skull fractures, should prompt a careful examination of the internal carotid 

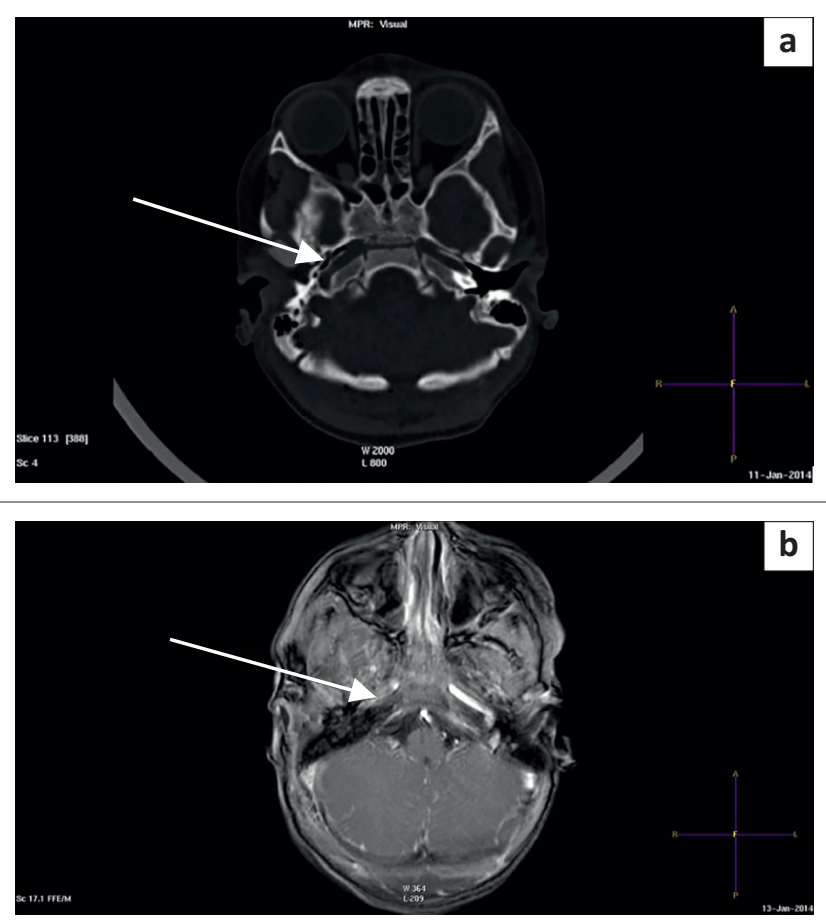

FIGURE 6: (a) A 5 year old boy presented with epistaxis, vomiting and a GCS of 12 , following blunt trauma. Axial CT bony algorithm demonstrated a right baseof-skull fracture, with air tracking along the right carotid canal (white arrow); (b) Axial T1 post contrast MRI of the brain shows a filling defect in the right petrous segment of the ICA (white arrow), in keeping with subacute thrombus secondary to dissection.

arteries (Figure 6a). ${ }^{3}$ In the absence of air but in the presence of a carotid canal fracture, we recommend proceeding to MRA swiftly in order to exclude carotid artery dissection. In order to avert additional radiation, MRA is preferable to CTA (Figure 6b).

\section{Hunting high and low Bridging vein thrombosis}

In known or suspected abusive head trauma/shaken baby syndrome (SBS) it is imperative to look for multifocal or subdural haematomas in different stages of breakdown, either with or without cerebral injury.

We would, however, stress the need to carefully screen the vertex (hunt high) to look for bridging vein thrombosis, particularly when associated with an acute subdural haematoma. The features of SBS are exhaustively described in the literature but little mention is made of bridging vein thrombosis, which is an important finding and of high diagnostic value for SBS. ${ }^{4}$ These veins lie within the subarachnoid space and constitute a short, non-tortuous, perpendicular pipeline between the arachnoid and the dura. ${ }^{4}$ They are especially vulnerable to rupture during movement in the antero-posterior direction (violent shaking). Thrombosis occurs within a few hours. ${ }^{4}$

A case in point was a five-month-old child who was referred with failure to thrive and seizures. A chest radiograph showed multiple rib fractures. This prompted
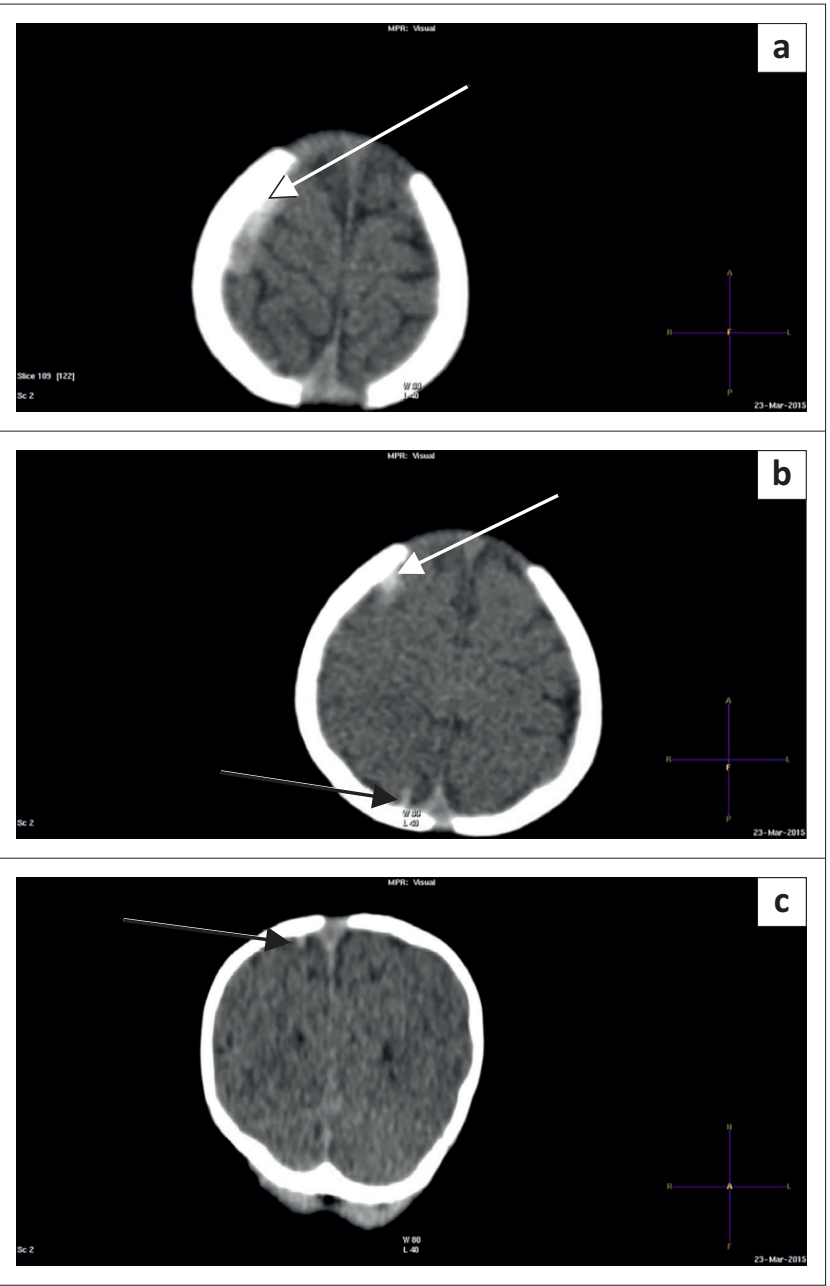

FIGURE 7: (a) A 5month old infant presented with failure to thrive, and new onset seizures. Axial CT brain demonstrated an acute right frontal subdura haematoma (white arrow); (b) Tubular hyperdensity in the right high parietal lobe, consistent with a cortical vein thrombosis (black arrow). The acute right lobe, consistent with a cortical vein thrombosis (black arrow). The acute right
frontal subdural haematoma (white arrow) is also demonstrated on this slice; (c) Coronal MPR CT brain confirms the high /vertex location of the bridging vein thrombosis (black arrow)

an emergency CT head, which demonstrated bridging vein thrombosis and an acute subdural hematoma, which assisted in confirming the diagnosis of non-accidental injury (Figures 7a-7c).

\section{Retroclival haematoma}

Retroclival haematoma is a paediatric entity that occurs with trauma. This lesion may easily be missed if not specifically looked for (hunt low) as this haematoma may be either a sliver, along the clivus, or large, resulting in brainstem or cord compression.

Reportedly $20 \%$ of patients with atlanto-occipital injuries may initially present with intact neurology. Another important point to remember is that the cervical spine plain film or bony sagittal cervical spine reconstruction may be normal without any prevertebral swelling or bony injury.

The haematoma may be either epidural or subdural ${ }^{5}$ and has even been described as subarachnoid. ${ }^{6}$ The distinction may not be relevant, but suffice to say the epidural haematoma is below the tectorial ligament, which may or may not be intact, 

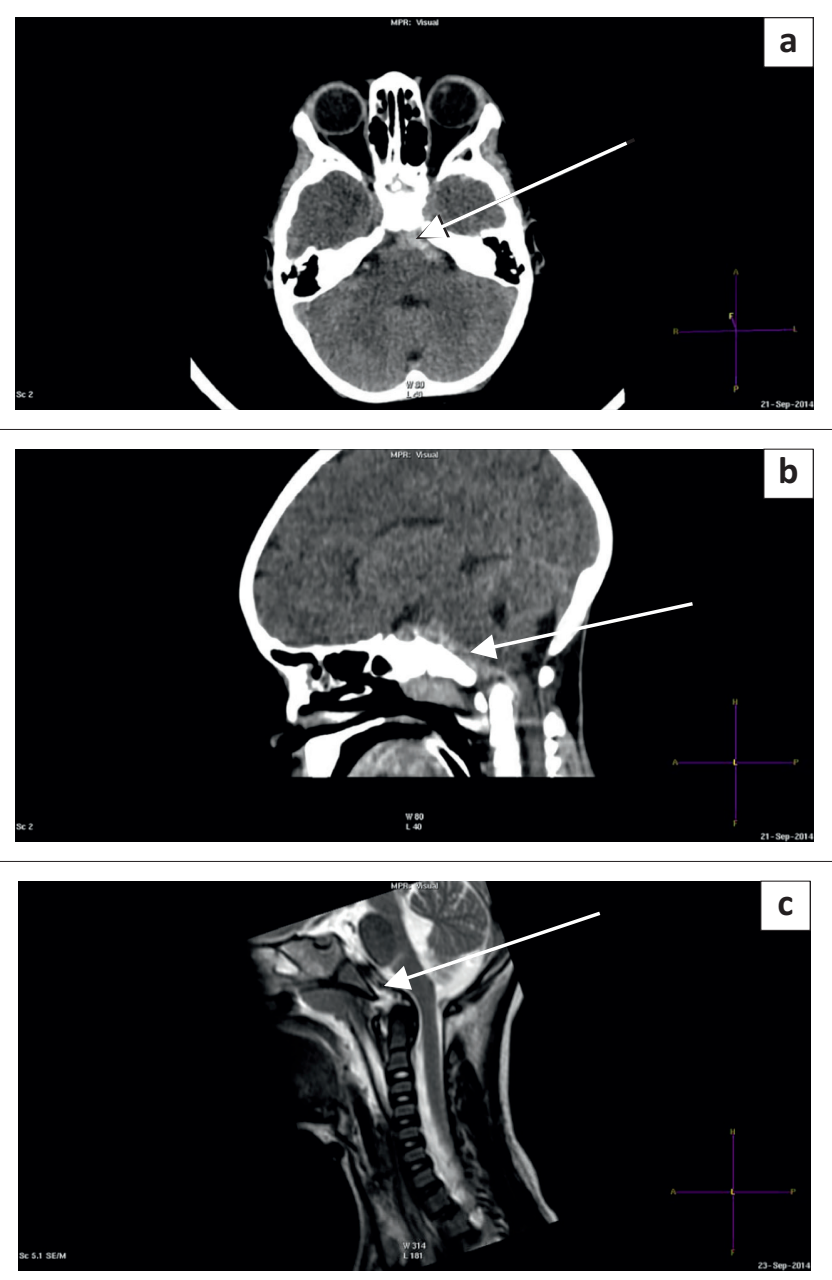

FIGURE 8: (a) A 6yr male involved in a high velocity MVA presented with decreased level of consciousness, hypertonia, decorticate posturing, and severe respiratory distress requiring intubation. Axial CT brain demonstrated a retroclival haematoma (white arrow); (b) Sagittal MPR CT brain confirms the retroclival location of the haematoma and extent (white arrow): (c) Sagittal T2 MRI brain confirmed a retroclival haematoma with tectorial ligament and probable apical ligament disruption (white arrow), with a normal proximal cord.

whereas the subdural haematoma lies on top of an intact tectorial membrane.

Screening of the cervical spine with MRI is necessary after identifying such a lesion to assess for associated ligamentous rupture (around the cranio-cervical junction) or associated cord injury, as these associations will determine the patient's management.

The trick is to have the pre-pontine (retroclival) space as a review area and always to review the brain in sagittal on the brain window algorithm so as not to miss this injury (Figures $8 a-8 c)$.

\section{Do not stop looking for lesions}

Severe head trauma, particularly that due to rotational acceleration and deceleration injury or shearing injury, can result in diffuse axonal injury (DAI). ${ }^{1}$ The history and mechanism of injury as well as poor clinical status will point to the diagnosis of DAI. Ideally, all patients should have an MRI for the purposes of prognosis, but in a resource-
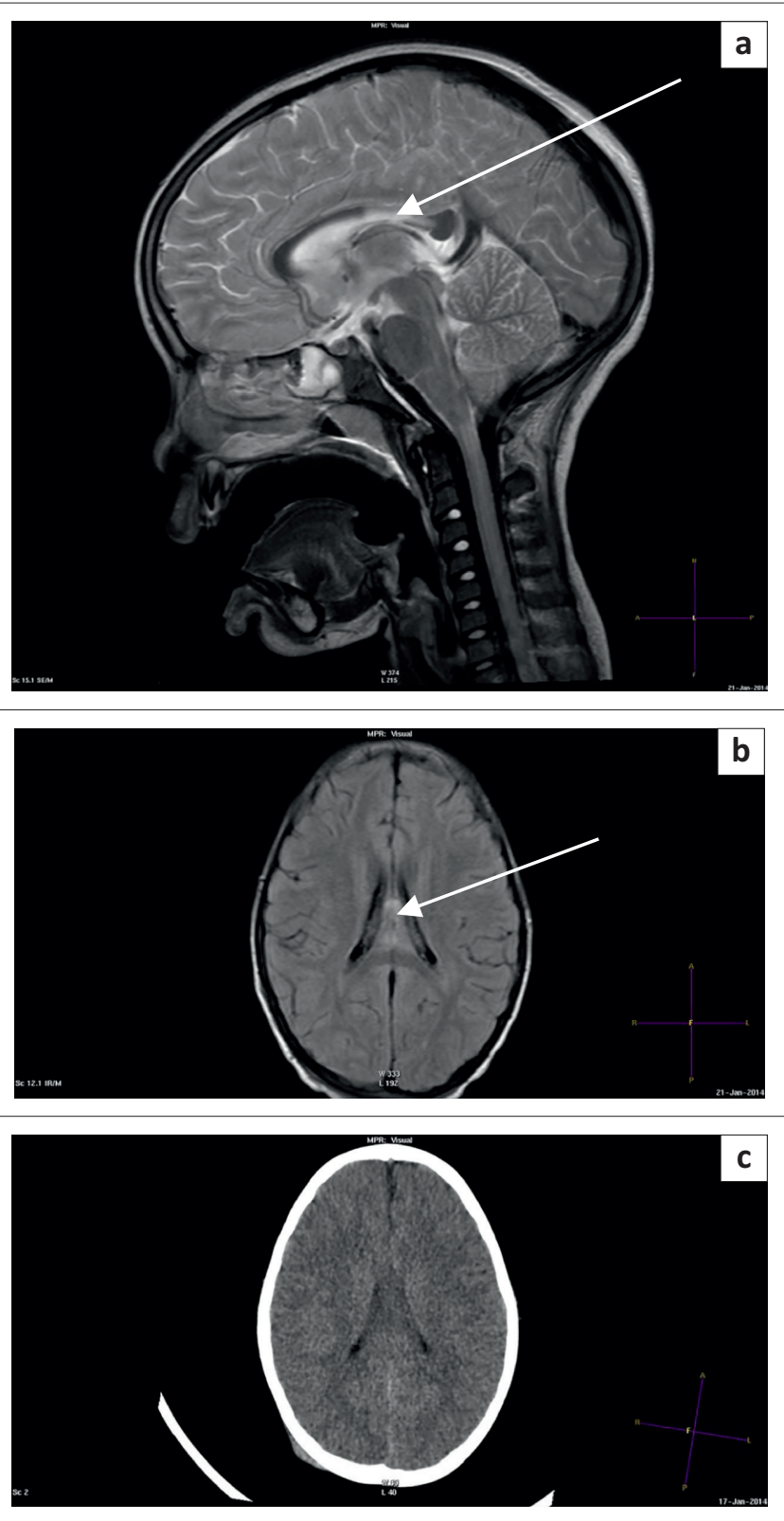

FIGURE 9: (a) A 9 year old girl presented with a head injury (GCS 8T), and suspected cervical spine injury. Sagittal T2 weighted MRI (large open field view) showed a corpus callosum shearing injury, with high signal in the body and extending to the splenium (white arrow). The cervical spine was normal; (b) Axial FLAIR MR confirmed the high signal in the body of the corpus callosum (white arrow); (c) Review of axial CT brain on admission showed no features of callosal injury.

poor setting this is not always feasible. Often CT does not appreciate all the lesions, particularly those affecting the corpus callosum, which are often missed on CT.

Over the years we have noticed a trend in requests for 'hemiplegia' or 'not moving a limb' in these severe head injury patients. MRI spine requests are often made in the face of severe head trauma in order to look for a cord injury. If there are no time constraints and an anaesthetist is readily available; then a complete brain and spine MRI is ideal. However; where time is an issue; we have found that a large field of view T2 sagittal of the cervical spine and including the corpus callosum is extremely useful. Often patients with suspected cervical spine injuries have corpus callosum shearing injuries and a normal cervical cord (Figures 9a-9c). 
This can thus limit the investigation to a 'brain only' scan.

\section{Conclusion}

Radiologists play a vital role in the interpretation of images related to paediatric head trauma. It is important to remember to review areas so as not to miss pathology that may affect the outcome and management of paediatric patients.

\section{Acknowledgement}

Thank you to Lyle Eyssen for assistance with reproducing the images.

\section{Competing interests}

The authors declare that they have no financial or personal relationships which may have inappropriately influenced them in writing this article.

\section{Authors' contributions}

R.R. (Groote Schuur Hospital) and N.W. (Red Cross War Memorial Children's Hospital) contributed equally to the writing of this manuscript.

\section{References}

1. Bhatt AA, Hunsaker J, Kalina P. Pearls and pitfalls of pediatric head trauma imaging. Appl Radiol. 2014 [cited 2015 Apr 17]. Available from: http://www. appliedradiology.com

2. Peedikayil T, Wieselthaler N. Coronal views of the paediatric mandibular condyle on computerised tomography brain (CTB) studies: An important review area. S Af J Rad. 2013;17(1):38-39. http://dx.doi.org/10.4102/sajr.v17i1.211

3. Orman G, Tekes A, Poretti A, Robertson C, Huisman TA. Posttraumatic carotid artery dissection in children: Not to be missed! J Neuroimaging. 2014;24(5):467-472. PMID: 24251954, http://dx.doi.org/10.1111/jon.12071

4. Adamsbaum C, Rambaud C. Abusive head trauma: Don't overlook bridging vein thrombosis. Pediatr Radiol. 2012;42(11):1298-1300. PMID: 22885602, http:// dx.doi.org/10.1007/s00247-012-2434-y

5. Silvera VM, Danehy AR, Newton AW, et al. Retroclival collections associated with abusive head trauma in children. Pediatr Radiol. 2014:44(Suppl. 4):S621-631. PMID: 25501734, http://dx.doi.org/10.1007/s00247-014-3170-2

6. Koshy J, Scheurkogel MM, Clough L, Huisman TA, Poretti A, Bosemani T. Neuroimaging findings of retroclival hemorrhage in children: A diagnostic conundrum. Childs Nerv Syst. 2014;30(5):835-839. PMID: 24469948, http:// dx.doi.org/10.1007/s00381-014-2369-8 